\title{
IMAGES: a unique view of the galaxy mass assembly since $\mathrm{z}=1$
}

\author{
M. Puech ${ }^{1,2}$, F. Hammer ${ }^{2}$, H. Flores ${ }^{2}$, Y. Yang ${ }^{2}$, and B. Neichel ${ }^{2}$ \\ 1 ESO, Karl-Schwarzschild-Strasse 2, D-85748 Garching bei München, Germany \\ mpuech@eso.org \\ 2 GEPI, Observatoire de Paris, CNRS, University Paris Diderot; 5 Place Jules \\ Janssen, 92190 Meudon, France
}

\begin{abstract}
Summary. The Large Program IMAGES is near 2/3 of its completion. It provides us with kinematics (GIRAFFE deployable IFUs), gas chemistry (FORS2), detailed morphologies (HST/ACS) and IR photometry (Spitzer) for a set of 70 galaxies representative of intermediate mass galaxies $\left(M_{J} \leq-20.3\right.$ or $\left.1.5 \times 10^{10} \mathrm{M}_{\odot}\right)$ at $\mathrm{z}=0.4-0.75$. We discover that, 6 Gyr ago, a significant fraction of galaxies $(\geq 40 \%)$ had anomalous kinematics, i.e. kinematics significantly discrepant from those of rotational or dispersion supported galaxies. The anomalous kinematics cause the observed large dispersion of the Tully-Fisher relation at large distances. IMAGES will soon allow us to study distant galaxies at a level of detail almost comparable to that of nearby galaxies.
\end{abstract}

\section{Introduction: intermediate-mass galaxies}

It is now relatively well-established that $\sim 50 \%$ of the present-day stellar mass has been formed since $\mathrm{z}=1$. Most of this stellar mass has been formed in intermediate-mass galaxies $\left(3 \times 10^{10}\right.$ to $3 \times 10^{11} M_{\odot}$, i.e., $\sim \mathrm{L}^{*}$ galaxies $)$, as a result of strong star formation episodes during which galaxies take the appearance of luminous infrared galaxies (LIRGs, see [3]). However, it is still unclear what physical processes have driven this evolution. To this respect, internal kinematics of distant galaxies is a powerful tracer of the major processes governing star-formation and galaxy evolution in the early universe such as merging, accretion, and feedback related to star-formation and AGN. Robustly measuring the internal kinematics of distant galaxies is thus crucial for understanding how galaxies formed and evolved.

\section{A representative sample of intermediate mass galaxies}

A Large Program at VLT entitled IMAGES (Intermediate MAss Galaxy Evolution Sequence) is aiming to derive both resolved kinematics and integrated 
properties from VLT/GIRAFFE and FORS2 for galaxies selected in the Chandra Deep Field (CDFS), and to combine these observations with deep and high quality images from HST/ACS as well as with deep mid-IR photometry from SPITZER/MIPS. This Large Program has now reached about $2 / 3$ of its completion, and recently, 39 additional galaxies observed with GIRAFFE have been analyzed [9]. Combined with previous observations during the GTO [1], it leads to a sample of 74 galaxies, which represents so far the largest existing sample of resolved kinematics for distant galaxies. Because in IMAGES we have deliberately selected $M_{J}(\mathrm{AB})<-20.3$ galaxies (i.e., with stellar masses larger than $\left.1.5 \times 10^{10} \mathrm{M}_{\odot}\right)$, we assume a similar limit for the combined sample. It let us with a sample of 63 galaxies which is well representative of the luminosity function at $\mathrm{z}=0.4-0.75$. Notice that the combined sample includes galaxies from 4 independent fields of view [9] and is then unaffected by field-tofield variations within Poisson statistics. Within this redshift range, GIRAFFE is able to recover the kinematics of almost all galaxies with $W_{0}([\mathrm{OII}]) \geq 15 \AA$.

\section{Kinematics of distant galaxies}

At large distances the spatial resolution is not sufficient to resolve the central regions of the galaxies. It implies that the observed velocity dispersion $(\sigma)$ of a rotational body is the convolution of the actual random motions with the rotation. For a rotationally supported galaxy, it unavoidably leads to a well defined peak in the centre (see Fig. 1). We have developed a classification scheme which allows us to compare any dispersion map to what it could be if it was a rotational disk [1]. Discrepancy from a rotational body can be measured from differences in amplitude and in position of the $\sigma$ peak between the pseudo rotational $\sigma$ map and the observed one, which leads to a very robust diagnostic diagram for the 63 observed galaxies [9].

Among the 63 galaxies of the representative sample, we find 20 rotating disks (32\%), 16 rotating disks with perturbations (25\%) and 27 galaxies with complex kinematics (43\%, see Fig. 1 and [9]). Within this classification, perturbed rotations correspond to a discrepant $\sigma$ peak from expectations for a pure rotation. The complex kinematics class corresponds to objects for which the large scale motions are not aligned to the optical major axis, and show $\sigma$ map very discrepant from expectations for a rotation (see Fig. 1).

\section{Comparison with morphology}

Among these 63 galaxies, 52 have multi-band HST/ACS imaging. Neichel et al. (in prep.) have constructed a new procedure to classify the morphology of distant galaxies. This procedure relies on two important ingredients which are the used of color maps (which helps to overcome limitations due to k-morphological corrections) and the use of a visual decision tree. This 


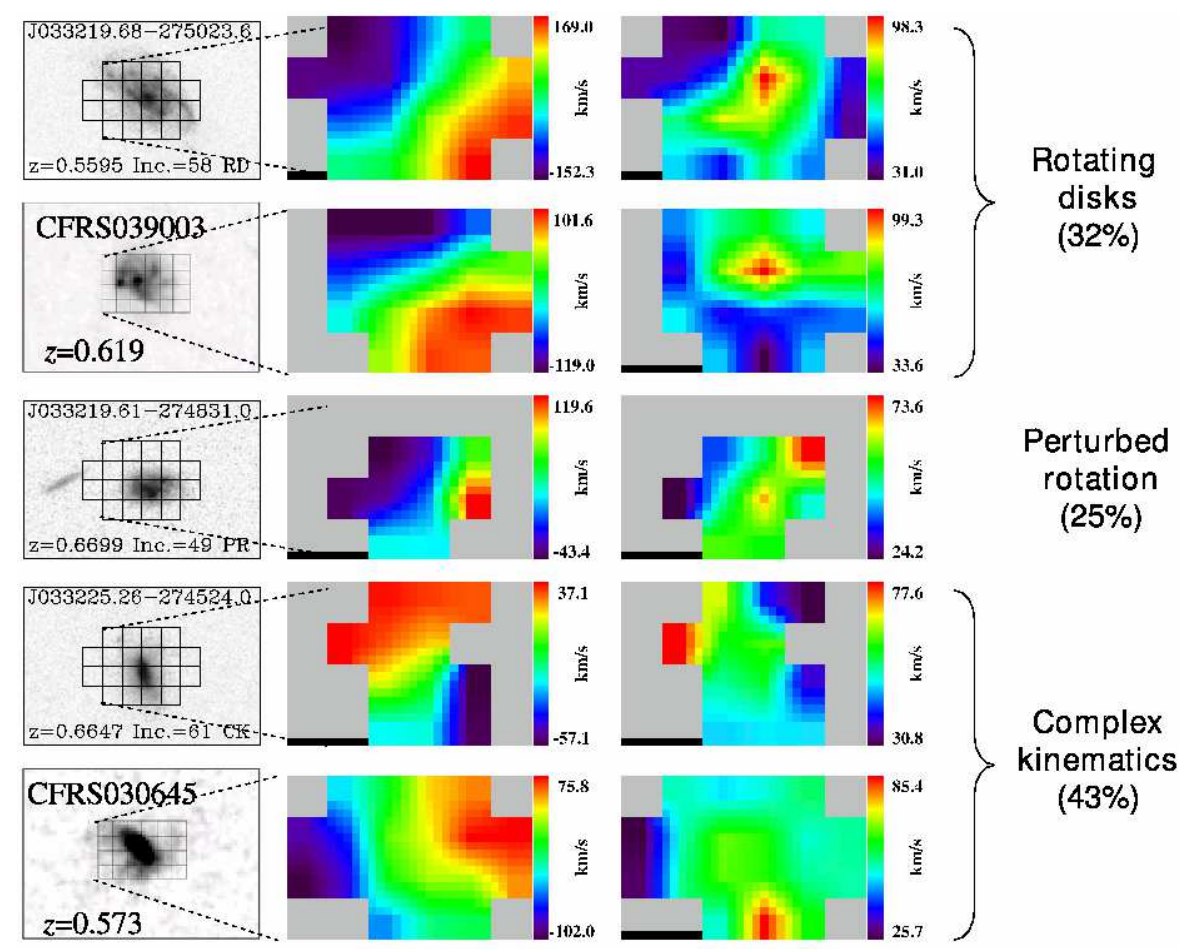

Fig. 1. Examples of kinematics of $\mathrm{z}=0.4-0.75$ galaxies; Each row corresponds to one galaxy. From left to right: HST ACS F755W/F814W image, observed velocity field and $\sigma$-map. The two top rows show regular rotating disk; the three bottom rows show galaxies with anomalous kinematics, one with just a shift of the peak in the $\sigma$-map, e.g. a perturbed rotation, the two other with dynamical axis misaligned relatively to the main optical axis.

last ingredient is particularly important, as they have shown that automatic methods can overestimate the fraction of spirals by a factor of $\sim 2$. They find a relatively good agreement between the two classifications: most of Rotating Disks are classified as Spirals, while most of galaxies having Complex Kinematics show a peculiar morphology.

Combining morphology with spacially resolved kinematics allows us to study distant galaxies in very fine details: for the first time, we have recently detected a minor merger with a mass ratio of $\sim 1: 18$ in a $\mathrm{z} \sim 0.6$ galaxy classified as a perturbed rotator [7] (see Fig. 2). Such processes, which are predicted to be much more numerous than major mergers by numerical models, could provide us with a very plausible mecanism for explaining the kinematics of perturbed rotators. 

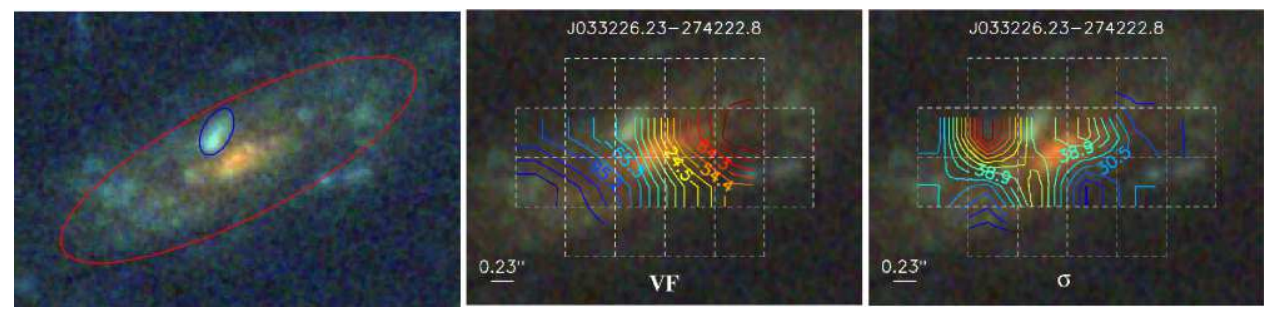

Fig. 2. Direct identification of a minor merger in a $\mathrm{z} \sim 0.6$ galaxy classified as a perturbed rotator [9]. Left: 3-band B-V-z HST/ACS image, with the blue ellipse indicating the position of the infalling satellite. Middle: 3-band image with GIRAFFE isovelocities superimposed. The GIRAFFE IFU bundle is shown in white dashedlines. Right: 3-band image superimposed with GIRAFFE velocity dispersion map isocontours. The dispersion peak is shifted by one GIRAFFE pixel compared to the position of the stellar continuum of the satellite, which is due to shocks between the gas stripped out of the satellite during the interaction, and the gas of the main progenitor [6].

\section{Dynamical state of distant galaxies}

Let us now consider a representative sample of intermediate mass galaxies: at $\mathrm{z}=0.6,60 \%$ of galaxies have $W_{0}([O I I]) \geq 15 \AA[2]$. Let us assume that quiescent $\left(W_{0}([O I I])<15 \AA\right)$ galaxy are either rotationally or dispersion supported, thus minimising the fraction of galaxies with anomalous kinematics. Then our results imply that $42 \pm 7 \%$ of $\mathrm{z}=0.4-0.75$ galaxies have anomalous kinematics, including $26 \pm 7 \%$ possessing complex kinematics. Because up to $97 \%$ of local intermediate mass galaxies are either $\mathrm{E}, \mathrm{S} 0$ or spirals [3], it is likely that the fraction of anomalous kinematics is close to a few percents today. This leads to an extremely rapid evolution of kinematical properties of galaxies, with about 10 times more complex kinematics about 6 Gyr ago.

The observed evolution of the Tully-Fisher Relation (TFR) provides us with a strong and independent confirmation. It has been suggested that galaxies with non-relaxed kinematics (PR and CK) are responsible for the very large dispersions of the TFR at high redshift [1]. This result is confirmed by Puech et al. (in prep) using the new sample of 63 galaxies: all the dispersion of the distant TFR can be accounted for by galaxies having non-relaxed kinematics. So it is beyond doubt that kinematics is among the most rapidly evolving properties of galaxies.

Which physical process could explain such a dramatic evolution? Anomalous kinematics are linked with strong variations of the specific angular momentum consistent with a random walk evolution due to merging between galaxies, as predicted by the hierarchical scenario of galaxy formation [6]. Indeed, during a merger, and especially a major merger, galaxies pass through various stages during which the disk may be destroyed, generating significant discrepancies to the general behaviour of isolated rotating disks. 


\section{Conclusion}

We do find a strong evolution of the galaxy kinematics since $z=0.6$, with a significant fraction of galaxies with complex kinematics. Observations presented here tell us that major mergers could have played an important role in shaping galaxies as we observe them today. Indeed many estimates of the merger rate found that a typical intermediate mass galaxy should have experienced 0.5 to 0.75 major merger since $\mathrm{z}=1$ (see $[8,5]$ and references therein), and most of them since $z=2-3$. A scenario in which merging is the dominant physical process explains many evolutionary features since $z=1$, including the number density evolution of LIRGs and the emergence of galaxies with complex morphologies and with blue cores at $\mathrm{z}>0.4$ [3]. It is moreover particularly in agreement with the fact that the Milky Way has had an exceptionally quiet merger history [4]. Present-day $M *$ galaxies being mostly spiral galaxies, it is unlikely that they have all escaped a major merging since $z=2-3$.

\section{References}

1. Flores, H., Hammer, F., Puech, M., Amram, P., \& Balkowski, C. 2006, A\&A, 455, 107

2. Hammer, F., et al. 1997, ApJ, 481, 49

3. Hammer, F., Flores, H., Elbaz, D., Zheng, X. Z., Liang, Y. C., \& Cesarsky, C. 2005, A\&A, 430, 115

4. Hammer, F., Puech, M., Chemin, L., Flores, H., \& Lehnert, M. 2007, ApJ, 662, 322

5. Kartaltepe, J. S., Sanders, D. B., et al. 2007, astro-ph, arXiv:0705.2266

6. Puech, M., Hammer, F., Lehnert, M. D., \& Flores, H. 2007a, A\&A, 466, 83

7. Puech, M., Hammer, F., Flores, H., Yang, Y., Neichel, B., \& Rogrigues, M. 2007b, A\&AL, accepted, astro-ph/0711.0611

8. Rawat, A., Hammer, F., Kembhavi, A. K., \& Flores, H., 2007, ApJL, submitted

9. Yang, Y. B., Flores, H., Hammer, F. et al. 2007, A\&A, accepted, astroph/0711.2305 